\title{
Chamber transport of "foot" pulses for heavy-ion fusion
}

\author{
W. M. Sharp, D. A. Callahan-Miller, and M. Tabak \\ Lawrence Livermore National Laboratory, Livermore, CA 94550 \\ S. S. $\mathrm{Yu}$ \\ Lawrence Berkeley National Laboratory, Berkeley, CA 94720 \\ P. F. Peterson \\ University of California, Berkeley
}

\begin{abstract}
Indirect-drive targets for heavy-ion fusion must initially be heated by "foot" pulses that precede the main heating pulses by tens of nanoseconds. These pulses typically have a lower energy and perveance than the main pulses, and the fusion-chamber environment is different from that seen by later pulses. The preliminary particle-in-cell simulations of foot pulses here examine the sensitivity of the beam focusing to ion-beam perveance, background-gas density, and pre-neutralization by a plasma near the chamber entry port.
\end{abstract}

\section{INTRODUCTION}

In a typical indirect-drive inertial-fusion target, the fuel capsule is heated and compressed by $\mathrm{X}$ rays from a metal shell, or "hohlraum"[1]. Hohlraum heating is done in two stages in order to produce correctly timed shock waves in the capsule. The hohlraum is first heated by a "foot" pulse to about $100 \mathrm{eV}$ and maintained at that temperature for around $25 \mathrm{~ns}$, then a second influx of energy raises the hohlraum temperature above $200 \mathrm{eV}$. For targets heated by heavy-ion beams, current designs $[2,3]$ require foot pulses to deliver $15 \%$ to $30 \%$ of the $3.3-6 \mathrm{MJ}$ total energy.

Numerical simulations of chamber transport have to date modeled only the main pulse[4-6]. However, simulations of foot pulses are also needed, due to differences both in beam parameters and in the chamber environment. Foot beams, in present designs, have about $25 \%$ lower ion energy than main pulses to compensate for range shortening in heated materials. The average current of foot pulses is about a third that of main pulses, and their generalized perveance, loosely defined as the ratio of the edge potential of a beam to its kinetic energy[7], is about half. At this reduced energy, the cross sections for collisional stripping of the beam and ionization of the background gas are predicted to be about $25 \%$ higher than at the main-pulse energy. The chamber environment seen by foot pulses differs from that of the main pulse mainly in the absence of a photoionized plasma around the target. As the hohlraum temperature approaches $100 \mathrm{eV}$, it begins to emit soft $\mathrm{X}$ rays, which ionize the background gas near the target and help neutralize both the target and approaching ion beams[6]. However, photoemission is negligible while the hohlraum is being heated by the first 10-15 ns of a foot pulse, so this beam section must reach the target without added neutralization.

The present paper presents preliminary numerical modeling of a single foot pulse in a fusion chamber. The approximations made in the model are described in Section II, and results are presented in Section III for three plausible sets of beam parameters, focusing on the effects of beam current and species, background-gas density, and a pre-neutralizing plasma. Some tentative conclusions are offered in a final section.

\section{METHOD}

\section{A. Numerical Model}

An upgraded version of the two-dimensional electromagnetic particle-in-cell (PIC) code BICrz[8-10] is used here to assess transport of foot pulses in a target chamber. The code was written in 1991 by Langdon[9] to model chamber transport, and Callahan added simple models of stripping and background-gas ionization in 1995[4]. An unconventional feature of BICrz is its use of a spatially converging mesh to maintain adequate resolution as the beam compresses radially. In addition, the code has a relativistic particle advance, allows the use of multiple species, and operates under a Basis interpreter[11]. For the present work, BICrz has been retrofitted with an improved Monte-Carlo collisional-ionization model that allows multiple-electron beam-stripping events[12]. 
For the preliminary foot-pulse simulations presented here, we make several simplifications. Only a single beam is treated, ignoring possible inter-beam effects near the target, and the initial beam distribution function is highly idealized. The beam is assumed to be axisymmetric, with initially uniform density and emittance, and the current is constant except in rise and fall sections near the beam ends. Since BICrz allows only a four-sided computational domain, with uniform boundary conditions on each edge, we inject the beam through a conducting boundary where the chamber wall would be. No electron emission from conducting boundaries is permitted. The background gas in the chamber, resulting from the molten-salt jets planned for wall protection, is assumed to be initially uniform and neutral. The version of BICrz used here allows the gas to be only singly ionized by collisions with beam ions, and to save computation time, gas ions are immobile. Ion recombination processes and collisional scattering are also ignored here, approximations that are justified by the low background-gas density. Except for runs discussed in Section III E, the molten-salt jets themselves are neglected. Also, there is no metal boundary where the target would be, so any problems with target-charge build-up would not appear. Instead, we model the mirror symmetry of the target plane by placing a boundary at the nominal target location. This boundary is a symmetry plane for fields, and it absorbs ions while reflecting electrons. Finally, photoionization by soft $\mathrm{X}$ rays from the heated target is ignored, although this assumption is fully justified only during the first part of the pulse.

The collisional-ionization cross sections used here were calculated by Olson[12] using a Monte-Carlo technique. Electrons liberated by collisions are assigned a Maxwellian energy distribution with an average energy of $20 \mathrm{eV}$, approximating the distribution calculated by Olson, and their angular distribution is strongly peaked in the transverse direction. Specifically, electrons are given a Lorentzian angular distribution with a probability

$$
P(\phi)=\frac{\pi}{b} \frac{\arctan \left(b^{-1}\right)}{\left(\frac{2 \theta}{\pi}-1\right)^{2}+b^{2}},
$$

where $\phi$ is the polar angle relative to the beam direction, and the value $b=0.335$ gives a good fit to Olson's calculations. The velocity distribution is uniform in the azimuthal angle $\theta$. The Olson cross sections have not been verified experimentally due to the absence of heavy-ion sources near the expected energy and charge state. However, the values have been compared with results from a phenomenological model developed by Armel[13]. This comparison shows good agreement for beam ions with a charge state less than four, but widening discrepancies are seen for higher charge states. These differences are under investigation, although we expect that they do not seriously compromise the results, due to the small size of these higher-state cross sections. The two models also predict strikingly different beam-stripping cross sections for events producing two or more electrons. Nonetheless, simulations made with the two sets of cross sections give virtually the same focal spot, despite major differences in the final charge-state distribution of the beam.

\section{B. Parameters}

The foot-pulse parameters used here are based on the requirements of the two-sided distributed-radiator target design[2], developed at Lawrence Livermore National Laboratory. This kind of target requires 1.5 MJ to be delivered by the foot pulses, followed by 4.5 to $5 \mathrm{MJ}$ from the main pulses. To compensate for range-shortening as the target is heated, the energy of foot-pulse ions is $75 \%$ of the main-pulse ion energy. We note in passing that the foot current required by close-coupled targets[3] is about half of the value used here for each layout, but the hohlraum size is reduced by $27 \%$ and the required focal-spot dimensions are correspondingly reduced. This lower-current option should be investigated, but the tighter spot-size requirement make it more challenging.

The indirect-drive target described in Ref. 2 will work with ions having a wide range of masses, provided that the ions have approximately the same axial velocity and deliver the required total energy. However, for the same velocity, the kinetic energy $V$ of an ion is proportional to its mass $M$. If we write the total energy $E$ of a cluster of $N$ beams as

$$
E \approx N V I \Delta t
$$

where $I$ is the average beam current and $\Delta t$ is the beam duration, we see that the current scales like $M^{-1}$ when the number of beams and their duration are held constant. Also, the generalized perveance, given in SI units by

$$
\kappa \equiv \frac{1}{4 \pi \varepsilon_{0}} \frac{2 Z e I}{\gamma^{3} \beta^{3} M c^{3}}
$$


will vary like $M^{-2}$ under these conditions, where $Z$ is the average beam-ion charge state, $\beta$ is the average ion velocity scaled by the speed of light $c$, and $\gamma \equiv\left(1-\beta^{2}\right)^{-1 / 2}$ is the corresponding relativistic factor. From this scaling, it is clear that the choice of ion species involves a tradeoff. The cost of the accelerator is reduced if a lighter ion is used, due to the lower ion energy, but space-charge effects, which are roughly proportional to $\kappa$, complicate transport in the accelerator and in the fusion chamber.

In most of the foot-pulse simulations here, we use $\mathrm{Pb}^{+1}$ ions with a $3 \mathrm{GeV}$ energy, although runs with other species are discussed in Section IIIB. The initial beam density is assumed to be uniform, with a 30 -ns pulse duration and 6-ns parabolically varying rise and fall sections at the ends. The 18-ns middle section has a 3 -cm radius as it enters the fusion chamber, and the beam is focused at a point $3 \mathrm{~m}$ inside the chamber wall. The unnormalized edge emittance, defined here as

$$
\varepsilon_{\perp} \equiv 2\left[\left\langle r^{2}\right\rangle\left(\left\langle r^{\prime 2}\right\rangle+\left\langle r^{2} \theta^{\prime 2}\right\rangle\right)-\left\langle r r^{\prime}\right\rangle^{2}-\left\langle r^{2} \theta^{\prime}\right\rangle^{2}\right]^{1 / 2}
$$

is taken to be initially $9 \mathrm{~mm}$-mrad, where the angle brackets denote density-weighted radial averages, and the primes indicate derivatives of the particle transverse coordinates $r$ and $\theta$ with respect to the axial coordinate $z$. Results are not sensitive to this emittance value, however, due the sizable emittance growth during chamber transport. A molten salt composed of fluorine, lithium, and beryllium, referred to as "flibe", is frequently chosen to protect the walls in conceptual driver designs[14], and at the working temperature of $600^{\circ} \mathrm{C}$, this salt produces a vapor consisting of about $90 \% \mathrm{BeF}_{2}$ and $10 \% \mathrm{LiF}$. This composition is used in the simulations here. We specify a nominal vapor density of $5 \times 10^{13} \mathrm{~cm}^{-3}$, corresponding to about $3.1 \mathrm{mTorr}$, although recent work[15] suggests that the density may be as low as $7 \times 10^{12} \mathrm{~cm}^{-3}$. The effects of this possible lower density are discussed in Section IIIC.

The number of foot beams $N_{\text {foot }}$ is constrained by simple scaling considerations. If all beams are assumed to have the same charge $I \Delta t$ in the accelerator, as is desirable for efficient transport, then from Eq. (2), the number of foot pulses is

$$
\frac{N_{\text {foot }}}{N_{\text {main }}}=\frac{E_{\text {foot }}}{E_{\text {main }}} \frac{V_{\text {main }}}{V_{\text {foot }}}
$$

which gives a ratio of 0.44 for the values of $E$ and $V$ considered here. The same assumption also gives simple relations for the average current and perveance of foot pulses:

$$
\begin{gathered}
\frac{I_{\text {foot }}}{I_{\text {main }}}=\frac{\Delta t_{\text {main }}}{\Delta t_{\text {foot }}} \approx 0.33 \\
\frac{\kappa_{\text {foot }}}{\kappa_{\text {main }}}=\frac{\Delta t_{\text {main }}}{\Delta t_{\text {foot }}}\left(\frac{V_{\text {main }}}{V_{\text {foot }}}\right)^{3 / 2} \approx 0.51
\end{gathered}
$$

These scaling relations are, of course, somewhat flexible, since foot pulses could have less total charge than main pulses at a modest penalty in transport efficiency.

Geometric considerations further constrain the number of foot pulses. In the accelerator, the beams should be clustered in an approximately circular array to minimize the size of the induction cores, and the spacing between beams should be equal in the two transverse directions, since focusing is provided by quadrupoles. The best choice is therefore a square array with beams absent from corner positions to give the bundle an approximately circular shape. The array should be an even number of beams on a side so that it can be split down the middle to send equal numbers of beams to the two sides of the target. Also, the foot pulses should occupy outer columns in the accelerator, so they can be split off at a lower energy than the main pulses. Each beam array entering the fusion chamber should also be square with corner beams absent, but it should have an odd number of beams per to allow an opening at the center for target injection. To give a high degree of symmetry, the number of main pulses and foot pulses entering each side of the chamber should both be divisible by four, allowing four identical quadrants. Finally, a lower limit to the number of beams is imposed by the maximum perveance that can be transported in the chamber, while an upper limit is set by practical component sizes and by the overall complexity, although both limits may be relaxed by improved understanding and technology.

The 112-beam layout in Fig. 1 satisfies all of these constraints. Each half of the beams in the accelerator array in Fig. 1a can be rearranged to give the chamber grouping seen in Fig. 1b. For the 30-ns current profile described above, 
(a)

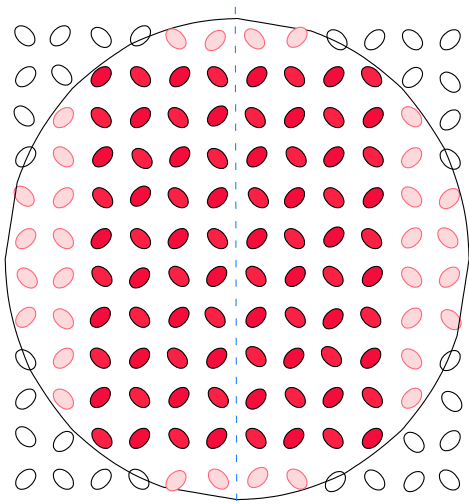

to left side to right side (b) chamber

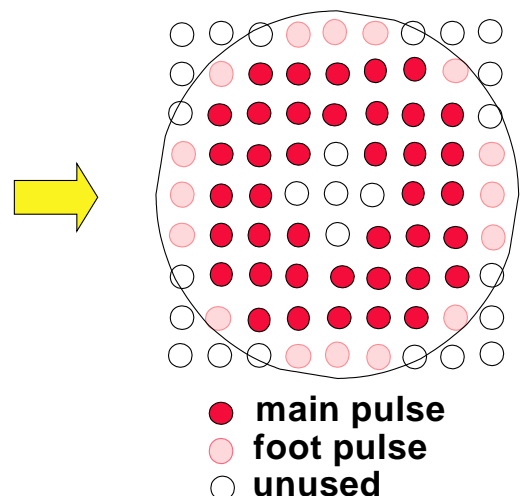

Figure 1: Sketches of beam layouts for 112-beam case (a) in the accelerator and (b) entering one side of the fusion chamber.

(a)

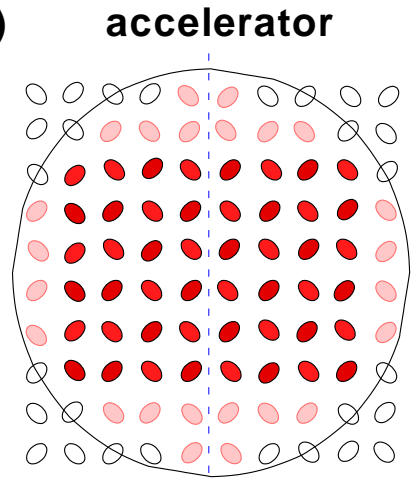

to left side to right side (b) chamber

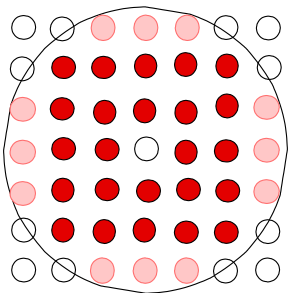

main pulse

foot pulse

unused

Figure 2: Sketches of beam layouts for 72-beam case (a) in the accelerator and (b) entering one side of the fusion chamber.

(a)

accelerator

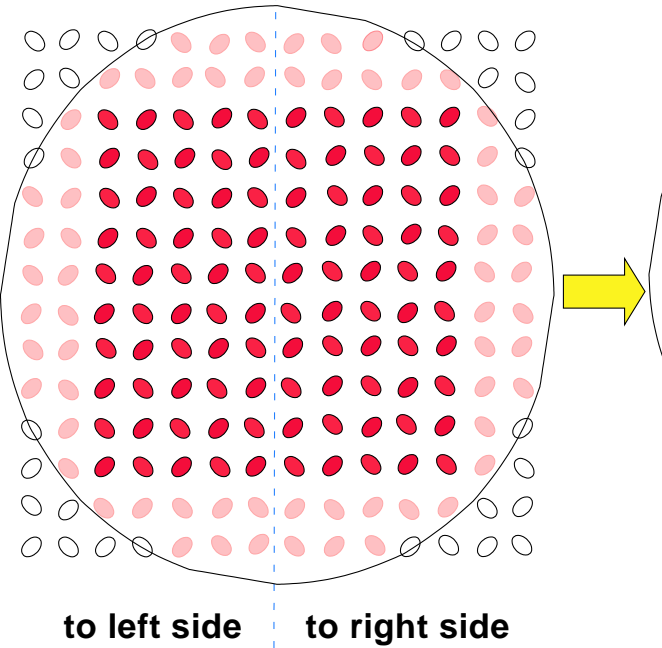

(b) chamber

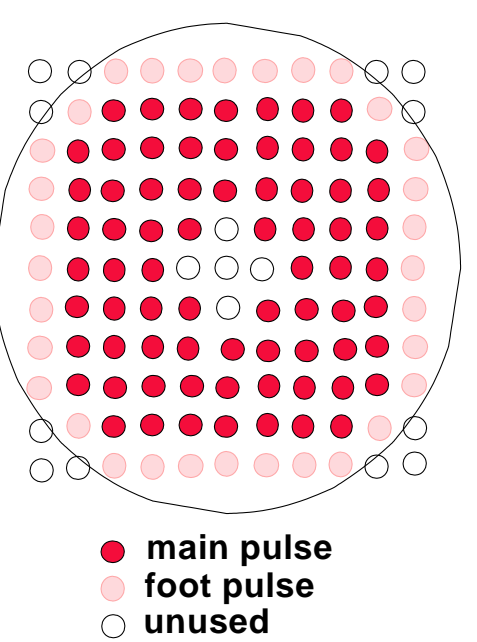

Figure 3: Sketches of beam layouts for 204-beam case (a) in the accelerator and (b) entering one side of the fusion chamber. 

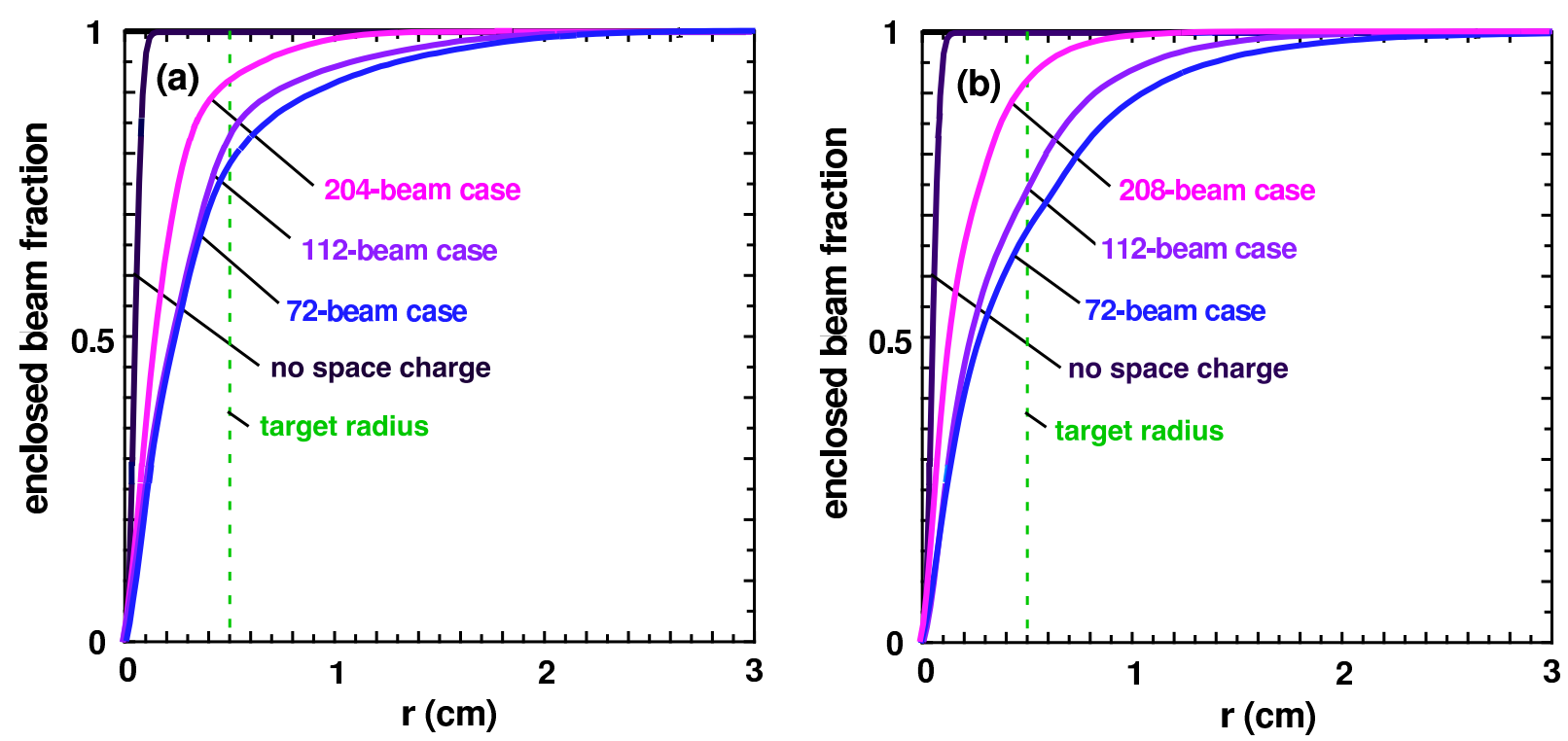

Figure 4: Enclosed fraction of beam ions at the beam waist for background-gas densities of (a) $5 \times 10^{13} \mathrm{~cm}^{-3}$ and (b) $7 \mathrm{x} 10^{12}$ $\mathrm{cm}^{-3}$, shown as a functions of radius for the three beam layouts treated here. Also, the enclosed-current fraction is shown for the same initial conditions but no space charge.

each of the 32 foot beams has a peak current of $651 \mathrm{~A}$, giving a generalized perveance of $3.6 \times 10^{-5}$. We shall take this as the nominal case. Decreasing the number of rows and columns by one in the chamber layout gives a 72 -beam case sketched in Fig. 2. Each of the 24 foot pulses in this case has a maximum current of 868 A, corresponding to a perveance of $4.9 \times 10^{-5}$. Similarly, adding another row and column to the nominal layout leads to the 208-beam case seen in Fig. 3. Here, there are 64 foot beams, each with a $325 \mathrm{~A}$ peak current and a $1.8 \times 10^{-5}$ perveance. Although cases with more or fewer beams might be worked out, these three cases span what seems currently practical.

\section{RESULTS}

\section{A. Effects of foot-pulse perveance}

The three cases presented above differ only in their initial beam current, so they illustrate the effects of varying perveance in a particularly simple way. A useful measure of chamber-transport effectiveness is the fraction of enclosed beam ions as a function of radius at some $z$-position, usually the point of best focus, called the beam "waist." This diagnostic measure is a time integral over the beam duration and is proportional to the energy that would be deposited by the beam if the target were placed at that $z$ location. Fig. 4a shows the enclosed beam fraction at the beam waist for the three beam layouts discussed above. Since the target radius is typically about $5 \mathrm{~mm}$, we see that between $8 \%$ and $23 \%$ misses the target end, with the amount decreasing with perveance, as expected. Also, the location of the waist shifts from about $2.75 \mathrm{~m}$ for the 72 -beam case to $3 \mathrm{~m}$ for the 208-beam case. The decreasing slope of the enclosed-fraction curves for larger radii indicates a decreasing density, caused by the blow-off of ions with a higher charge state. Without space charge, the same beams would focus to about a 1-mm spot, as seen in Fig. 4a, and would maintain a uniform density during compression, indicated by a quadratic variation of the enclosed-beam with radius.

The cause of this rather unsatisfactory focus is the emittance growth caused by the nonlinear radial electric field. As the beam compresses, the higher energy electrons near the beam edge are lost, leaving non-uniform neutralization and therefore a nonlinear radial electric field. Ionization of the background gas gives these beams an average neutralization fraction $f$ approaching about $90 \%$ after the first meter of propagation, as predicted by the analytic work of C. L. Olson[16]. However, this neutralization is largely offset by the increased beam charge due to stripping, which increases the average beam charge state in these cases to about five after three meters of transport, with a maximum charge state of eleven. The combination of these two effects is conveniently summarized by defining an "effective perveance" $\kappa_{\text {eff }} \equiv Z^{2}(1-f) \kappa_{0}$, where $Z$ is again the average charge state in a beam slice and $\kappa_{0}$ is the initial perveance from 

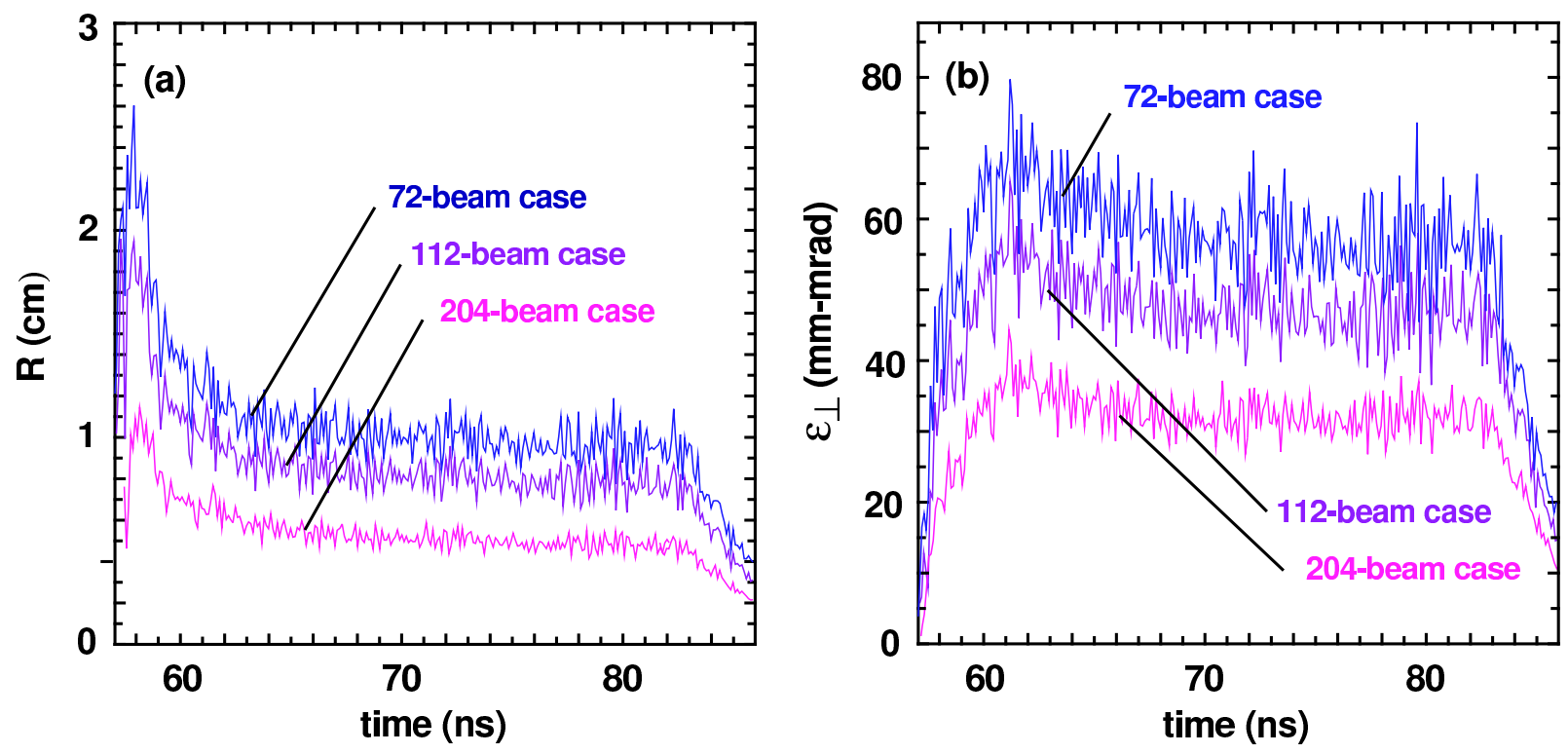

Figure 5: Time histories for three beam layouts of the beam (a) edge radius $R$ and (b) edge emittance $\epsilon_{\perp}$ after $3 \mathrm{~m}$ transport in background gas with the nominal density.
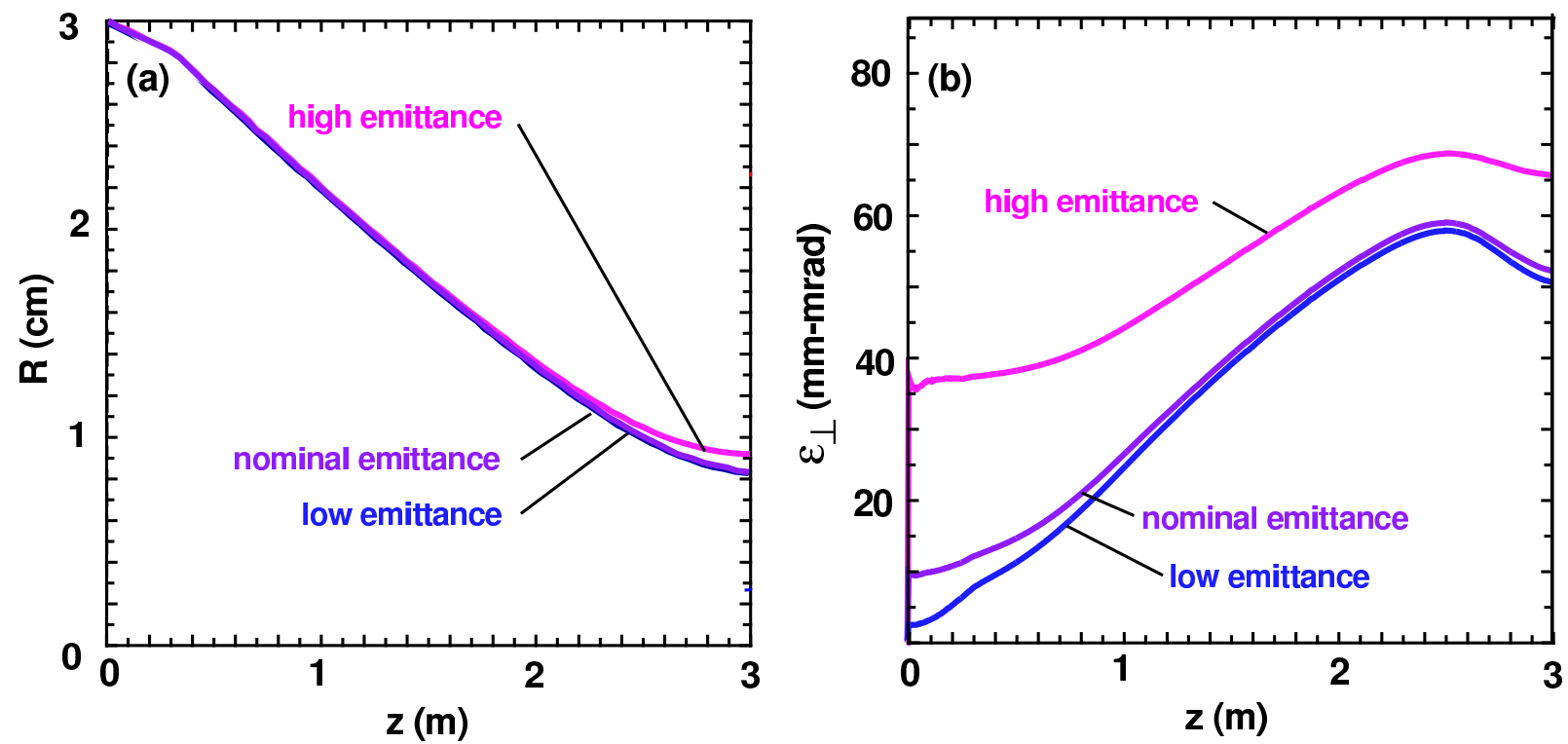

Figure 6: Histories of the (a) edge radius $R$ and (b) edge emittance $\epsilon_{\perp}$ of beam slices initially having the nominal emittance, one-quarter of that value ("low emittance"), and four times that value ("high emittance"). In each case, nominal parameters for the 112-beam case are used, and the background-gas density is $5 \times 10^{13} \mathrm{~cm}^{-3}$.

Eq. (3), assuming singly charged beam ions. We find that the effective perveance varies much less during transport than $\kappa$ itself, typically having an average value near $\kappa_{0}$. Consequently, the focal spots seen in Fig. $4 a$ are close to those seen in vacuum for the same parameters.

The poor focus evident in Fig. 4a is exaggerated by the head-to-tail variation in radius and emittance that develops during transport. The plots of the effective edge radius $R \equiv 2^{1 / 2}\left\langle r^{2}\right\rangle^{1 / 2}$ at the $3 \mathrm{~m}$ location in Fig. 5a show a pronounced growth of the beam head over the first $20 \%$ of the pulse for each case, which is reflected in the corresponding edge-emittance values of Fig. 5b. The radius growth near the head, which develops mainly during the last meter of transport, is a result of poorer neutralization in the first ionization length behind the beam head, corresponding to $13 \mathrm{~ns}$ in this case. Photoionization by $\mathrm{X}$ rays from the heated target is expected to increase head-to-tail variation, since the foot-pulse tail should be much better neutralized than then head. 

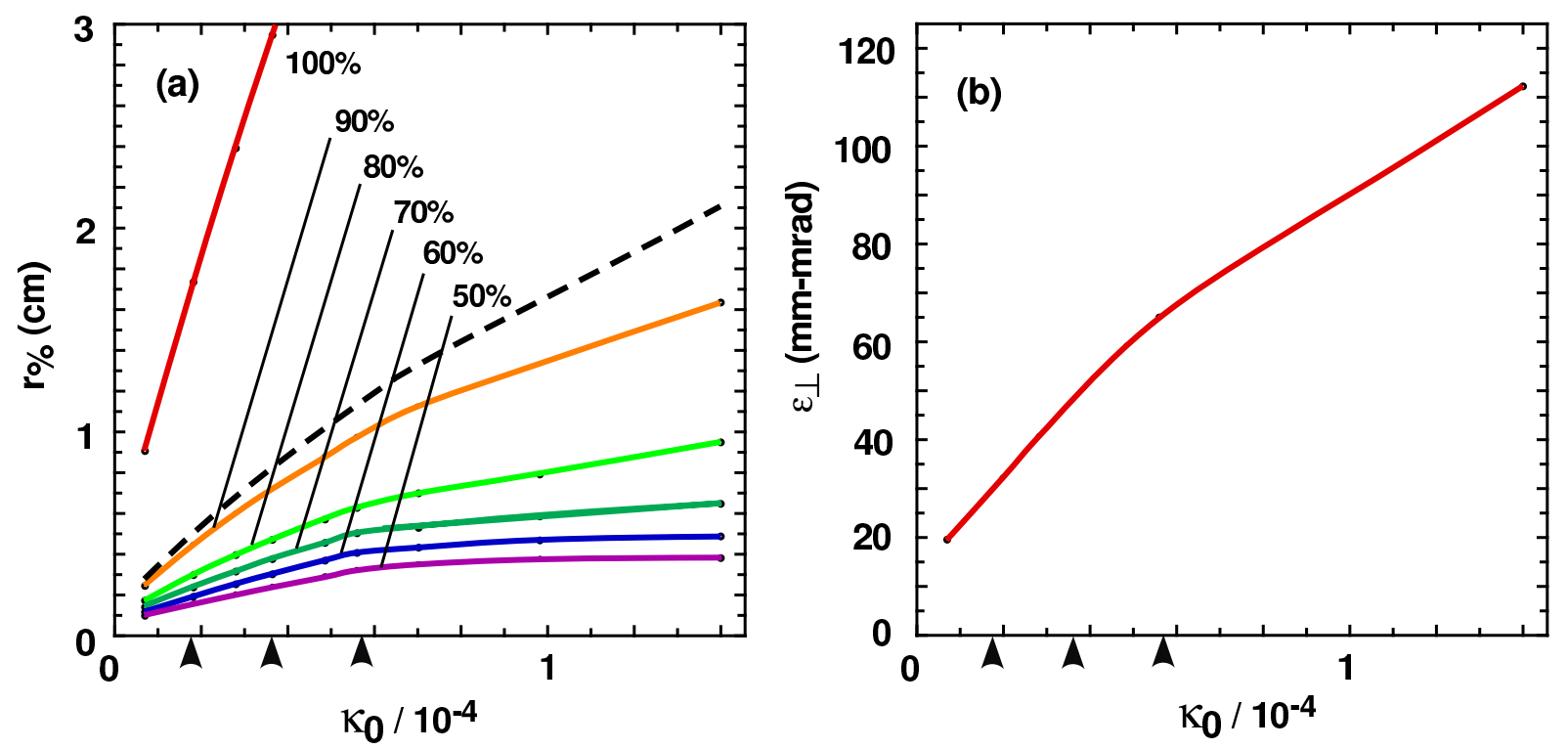

Figure 7: (a) Radii enclosing specified percentages of beam ions at the beam waist for the nominal $5 \times 10^{12} \mathrm{~cm}^{-3}$ background-gas density and (b) the corresponding edge-emittance values, both plotted as functions of the initial beam perveance. The dashed curve gives the effective edge radius $R$. Arrowheads mark the perveance values corresponding to the three beam layouts.

The focal spot shows little sensitivity to the inital emittance, so long as that value is small compared with emittance growth during chamber transport. Fig. 6 shows the change during chamber transport of the edge emittance $\epsilon_{\perp}$ and edge radius $R$ near the beam midpoint for three beams differing only in their initial emittance. The beams respectively have emittances of $2.25,9$, and $36 \mathrm{~mm}$-mrad. Otherwise, the nominal parameters for the 112-beam case are used. Values on the figure are obtained by averaging the emittance from Eq. (4) over $20 \%$ of the beam length near the midpoint, and they are plotted against the $z$ position of the midpoint. The slice thickness accounts for the inflections seen in the curves at $z=30 \mathrm{~cm}$, the point at which the entire slice has entered the computational domain. For the first two meters of transport, the emittance increase is well predicted by the analytic expression derived by Lee, et $a l .[17]$

$$
\epsilon_{\perp}(z) \approx\left[\epsilon_{\perp}^{2}(0)+\lambda^{2} \kappa_{e f f}^{2} z^{2}\right]^{1 / 2}
$$

where the coefficient $\lambda$ depends on the beam radial profile. In general, Eq. (8) has a term that is linear in $z$, but we can neglect it here because the fields of initial beam distribution vary linearly with $r$ inside the beam, so that $d \epsilon_{\perp}(0) / d z$ is zero. The calculated emittance growth departs from Eq. (8) near the waist because the expression was derived by assuming nearly ballistic ion trajectories, an assumption that fails near the minimum radius. The radius histories of the three cases are nearly identical until the final meter of transport, where they deviate to give minimum radii that are approximately proportional to the final emittance, as predicted by Ref. 8 .

The sensitivity of the beam focal spot to the initial beam perveance can be summarized by the radii $r_{\%}$ enclosing specified percentages of beam ions at the beam waist, plotted as functions of the initial perveance. Remarkably, the focal-spot data plotted in Fig. 7a indicated that the energy deposition pattern varies self-similarly over an order of magnitude of current values, as indicated by the nearly straight $r_{\%}$ lines. To a good approximation, the emittance at the waist, shown in Fig. $7 \mathrm{~b}$, is proportional to the effective radius $R$, as predicted by Ref. [17]. Nonetheless, emittance values from the simulations are typically about $30 \%$ smaller than the analytic prediction, due to the non-ballistic orbits near the target.

While perveance is an important determinant of chamber-transport effectiveness, beams with the same perveance may show significant differences in their dynamics. As seen in the definition of Eq. (3), perveance is determined by the beam-ion mass, velocity, and current, and these parameters affect beam dynamics is differing ways. For example, if the ion mass is decreased and the axial velocity is increased to give the the same perveance, the beam is more sensitive to nonlinearities in the radial electric field than the corresponding case with the nominal ion mass and will show a larger emittance increase. Likewise, simultaneously increasing the beam current and velocity while keeping the perveance constant leads to larger space charge and hence to greater emittance that the nominal case. 

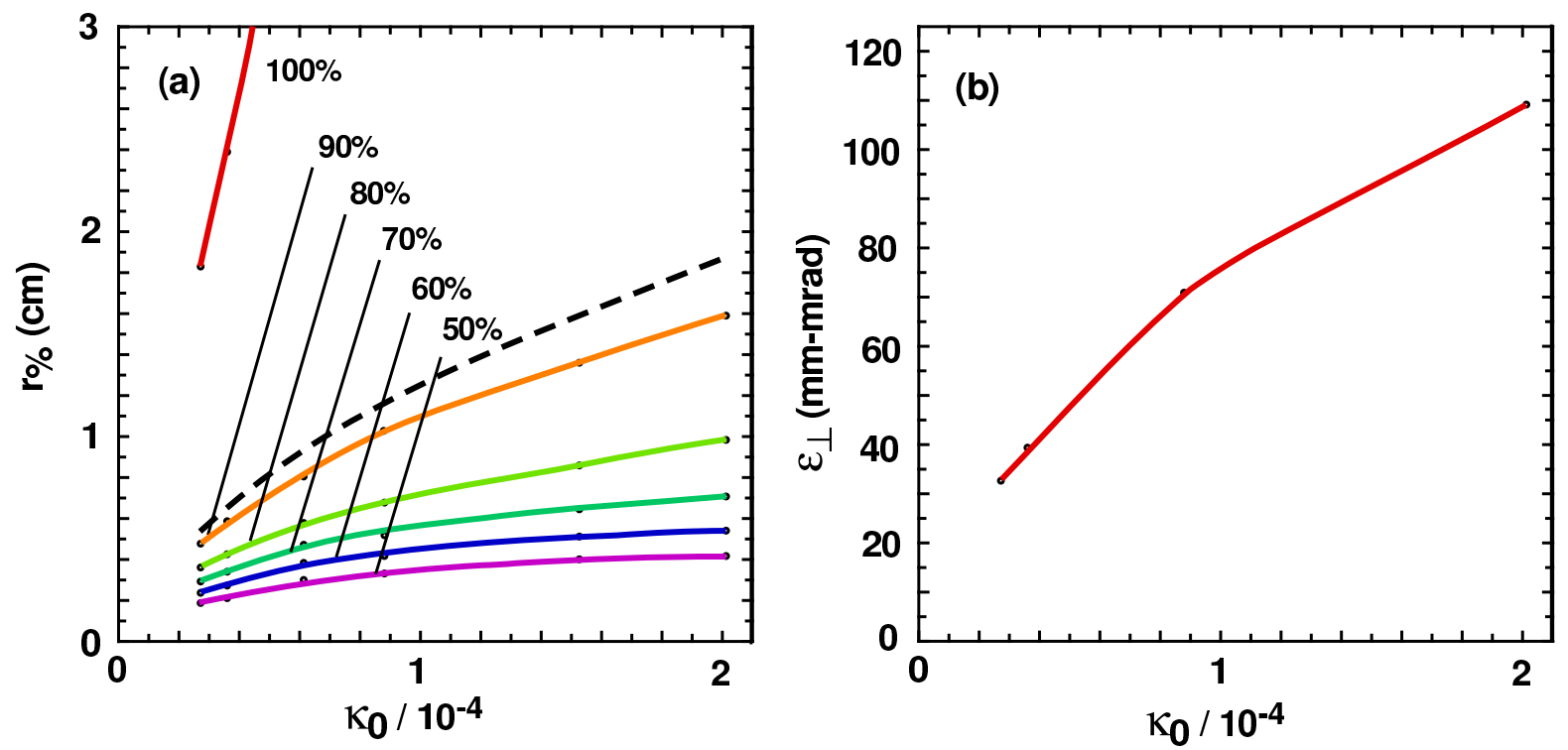

Figure 8: (a) Radii enclosing specified percentages of beam ions at the beam waist for six ion species and (b) the corresponding edge-emittance values, both plotted as functions of the initial perveance $\kappa_{0}$. The dashed curve gives the effective edge radius $R$.

\section{B. Effects of beam-ion species}

One method for reducing the cost of fusion driver is to use a lighter ion, which according to the scaling in Section II B, would require a proportionally reduced energy and would increase the perveance like the inverse square of the ion mass. The effects of using ions of different mass have been investigated in a series of simulations in which the deposited energy per beam and the ion velocity were held constant and the ion energy and current were adjusted appropriately. Six ion species with masses ranging from $88 \mathrm{amu}$ (strontium) to $238 \mathrm{amu}$ (uranium) were chosen, but the selection was based on their masses being well spaced rather than on the availability of ion sources. The total beam energy in each case was the same as the 112-beam case with $\mathrm{Pb}^{+1}$ discussed above, and we used the nominal $5 \times 10^{13}$ $\mathrm{cm}^{-3}$ background-gas density. The same collisional-ionization cross sections were used in every case, in part because calculations using the Armel model show less than $20 \%$ variation in cross sections over this mass range when the energy per nucleon is held constant, but also because we want to compare the focal-spot variation with with the results found in the previous section for current variation.

Focal-spot data for the five ion species and for lead are plotted in Fig. 8 versus perveance, which ranges from about 2 $\times 10^{-5}$ to $2.7 \times 10^{-4}$. Qualitatively, the results resemble those of Fig. 7. The beam focal spot increases self-similarly with perveance up to about $8 \times 10^{-5}$, and at larger perveance, the increase is less than linear. However, both the focal-spot size and the emittance vary more weakly with perveance than is seen when current is varied for a given species, even though the charge-state distribution and the transverse space-charge fields are quite similar for a given perveance. This difference arises because heavier ions are less responsive to the radial electric field and therefore spread out less as the beam converges.

\section{Effects of background-gas density}

Recent experimental work measuring the vapor pressure of molten flibe[15] indicates that the equilibrium density at the expected $600^{\circ} \mathrm{C}$ operating temperature may be substantially lower than previous estimates. Extrapolation from higher-temperature data had suggested the nominal $5 \times 10^{13} \mathrm{~cm}^{-3}$ value used in these simulations. The new data indicates that the actual density may be as low as $7 \times 10^{12} \mathrm{~cm}^{-3}$, corresponding to a pressure of about $0.4 \mathrm{mTorr}$.

Simulations with beam parameters for the three standard cases have been run using this lower density, and the focal-spot data are plotted in Fig. 4b. In each case, these runs give a slightly poorer enclosed-beam fraction as the 

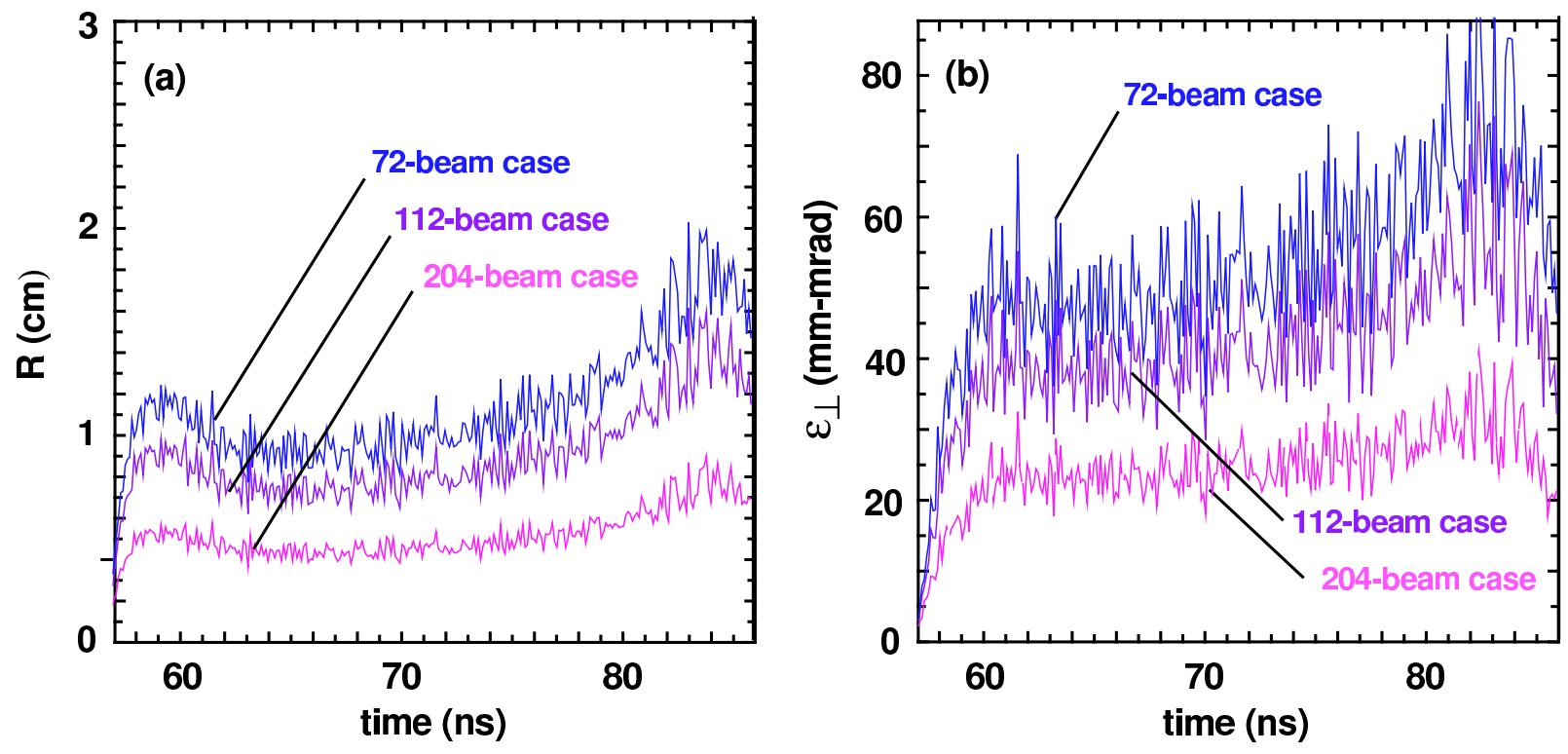

Figure 9: Time histories for the three beam layouts of the beam (a) edge radius $R$ and (b) edge emittance $\epsilon_{\perp}$ after $3 \mathrm{~m}$ transport in background-gas with a $7 \times 10^{12} \mathrm{~cm}^{-3}$ density.

corresponding nominal-density case, even though the emittance growth during transport is substantially smaller. The cause of this counter-intuitive result is the poorer neutralization at the lower density. At a gas density of $5 \times 10^{13} \mathrm{~cm}^{-3}$, the mean-free path for background-gas ionization is about $65 \mathrm{~cm}$, so after the beam has traveled about $1.3 \mathrm{~m}$, the neutralization fraction approaches a fairly steady value of about $95 \%$, with a small decrease due to electron heating as the beam compresses. At the lower density, however, the mean-free path exceeds $4.5 \mathrm{~m}$, and the neutralization fraction only reaches about $55 \%$. The final beam charge is much less at the lower density because collisional stripping gives an average charge state of 1.8 at the target, compared with 5.2 at the nominal density, but the lower neutralization leads to greater radial electric fields and emittance growth. The final $\kappa_{e f f}$ for the lower density is slightly greater than for the nominal density, which is consistent with the poorer focus.

Although the calculated focal spot shows very little variation over the expected range of background-gas densities, both the final beam emittance and the head-to-tail beam-radius variation show much more sensitivity over this density range. Figure 9 shows final edge radius and emittance values as functions of time for the three layouts studied here. Comparing with corresponding plots at nominal density in Fig. 5, we see that the midpoint radius values for the two densities are very similar, and the emittance values there differ by only about $15 \%$. However, the poor head confinement found at nominal density is not evident, and instead the largest radius and emittance values are seen near the beam tail. This difference results from the migration of electrons toward the head when the beam is poorly neutralized. Due to beam convergence, the beam head has a smaller radius and higher charge density than the tail, and provided that free electrons are effectively collisionless, as is the case over the range of densities studied here, they are attracted by the space-charge field. At the nominal density and above, the entire beam quickly reaches its asymptotic neutralization, so this head-to-tail charge difference does not develop. The tail bulge seen in Fig. 9 disappears gradually with decreasing background-gas density as the number of free electrons drops.

The series of runs plotted in Fig. 10a shows that, in the absence of pre-neutralization, the minimum waist occurs near the density at which the mean free path for collisional ionization approximately equals the fusion-chamber radius. For the parameters used here, this density is about $10^{13} \mathrm{~cm}^{-3}$. Although the edge radius, shown as a dashed line, is quite insensitive to pressure over the range studied, it is clear from the spacing of contours that the density profile within the beam is changing substantially. At higher gas densities, the core of the beam remains reasonably well-pinched while the outer $20 \%$ spreads to progressively larger radii. Below about $10^{13} \mathrm{~cm}^{-3}$, however, the spacing of beam-fraction lines becomes nearly quadratic, reflecting a flatter interior density profile. As the density is further reduced, the lines asymptote to their vacuum values, a few percent higher than the values shown. In this zero-pressure limit, the transverse density profile remains flat all the way to the target. 

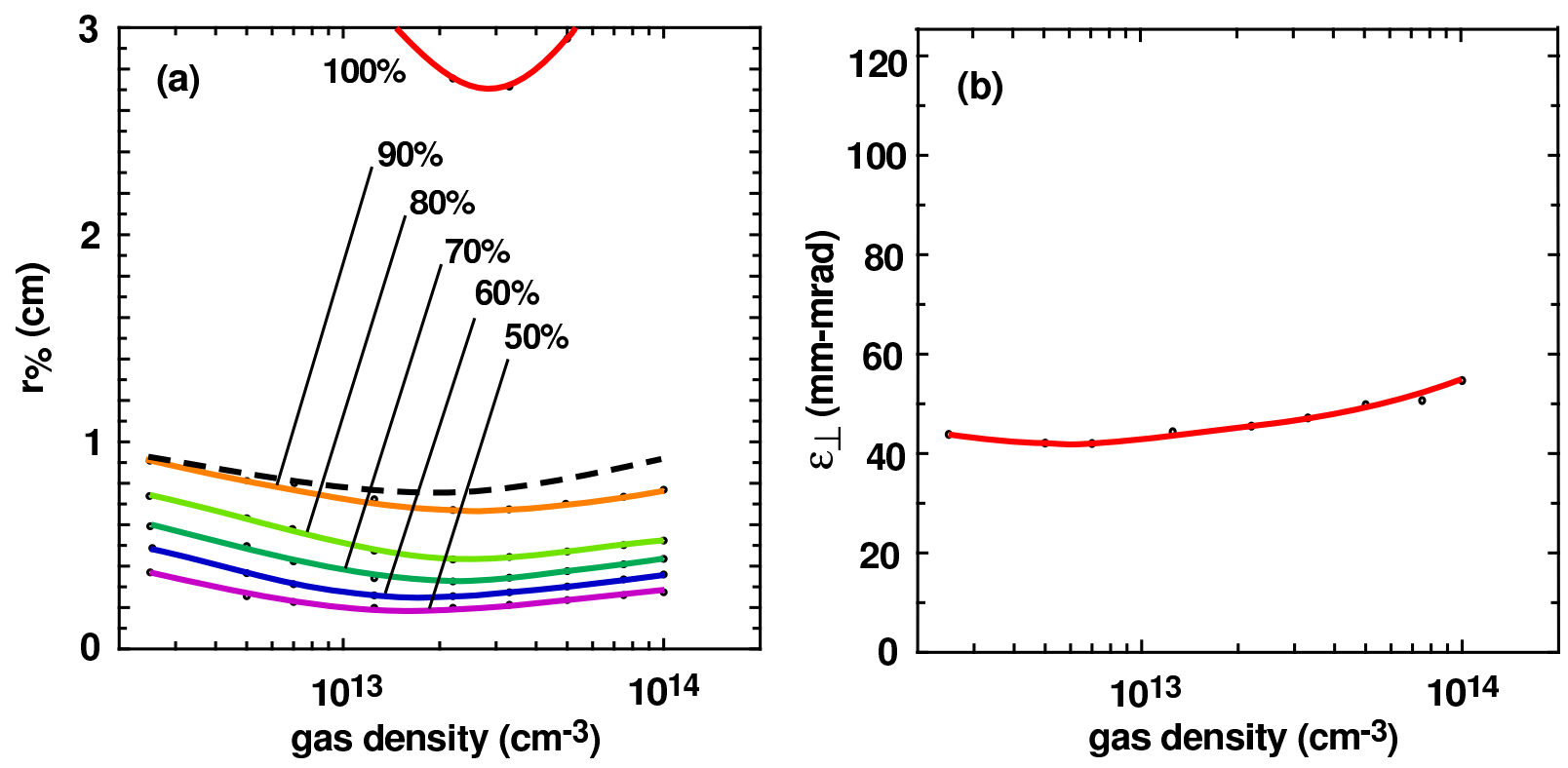

Figure 10: (a) Radii enclosing specified percentages of beam ions at the beam waist and (b) the corresponding edge-emittance values, shown as functions of the background-gas density. The dashed curve gives the effective edge radius $R$.

\section{Effects of pre-neutralization}

One suggestion for improving chamber transport is to pre-neutralize the beam by passing it through a fully ionized plasma[18]. The plasma would likely be introduced upstream from the chamber in a beam pipe, where image forces and wall emission would help maintain plasma quasineutrality as electrons are extracted by a beam. However, limitations in BICrz make realistic simulations of this configuration difficult. Since BICrz presently has non-emitting boundaries and allows only a topologically cylindrical computational domain, we ignore the beam pipe and place the neutralizing plasma $25 \mathrm{~cm}$ inside the chamber wall. Also, to minimize beam stripping, the best pre-neutralizing plasma would be fully ionized hydrogen. However, the mass of hydrogen is about one amu, compared with $47 \mathrm{amu}$ for $\mathrm{BeF}_{2}$, making the assumption in BICrz of fixed background ions questionable. For the cases described here, the plasma is cylindrical, with a radius of $4.5 \mathrm{~cm}$ and a length of $25 \mathrm{~cm}$, and it has a uniform density of $10^{12} \mathrm{~cm}^{-3}$.

For the nominal $5 \times 10^{13} \mathrm{~cm}^{-3}$ background-gas density, this pre-neutralizing plasma is found to make only a small improvement in the minimum beam radius. Plots of enclosed-beam fraction versus radius at the beam waist are shown in Fig. 11 for pre-neutralized beams at the nominal density. Comparing these with data in Fig. 4a for beams without pre-neutralization, we see that the current falling within the $0.5 \mathrm{~cm}$ target radius increases by at most $5 \%$ for the three standard beam layouts. This difference is due to improved neutralization inside and just following the plasma, but at the nominal background-gas density, the change is small. Without a plasma, the beam neutralization fraction $f$ approaches a steady value after a transport distance approximating twice the characteristic length for background-gas ionization, a total distance of $1.3 \mathrm{~m}$ in flibe with a $5 \times 10^{13} \mathrm{~cm}^{-3}$ density. This pattern is seen in the Fig. 12a plot showing $f$ near the midpoint of a 651-A beam without pre-neutralization. At this density, collisional ionization alone neutralizes about $75 \%$ of the beam charge by the end of the plasma layer, so an exiting beam pulls away relatively few plasma electrons. Consequently, after the plasma, Fig 12a shows little difference in $f$ with and without pre-neutralization. Halfway to the target, both beams are near the maximum neutralization value predicted by C. L. Olson [16].

Pre-neutralization to found to work much better at lower gas density. At a gas density of $7 \times 10^{12} \mathrm{~cm}^{-3}$, the enclosedfraction plots in Fig. 11b show dramatically better focus for each of the three layouts considered here, compared with the corresponding plots in Fig. $4 \mathrm{~b}$ without pre-neutralization. The fraction of current falling within a $5 \mathrm{~mm}$ radius increases from $62 \%$ to $86 \%$ for the 72 -beam case, while the 208-beam case shows an increase from $92 \%$ to $98 \%$. In addition, the pre-neutralized cases show less sensitivity to beam perveance than those without pre-neutralization, and the time-averaged emittance at the target location for each case is reduced by more than $40 \%$. The cause of this improvement is seen in Fig. 12b, showing the mid-pulse neutralization fraction for a 651-A beam in gas with a density of $7 \times 10^{12} \mathrm{~cm}^{-3}$. With pre-neutralization, the beam emerges from the plasma with $60 \%$ neutralization, compared 

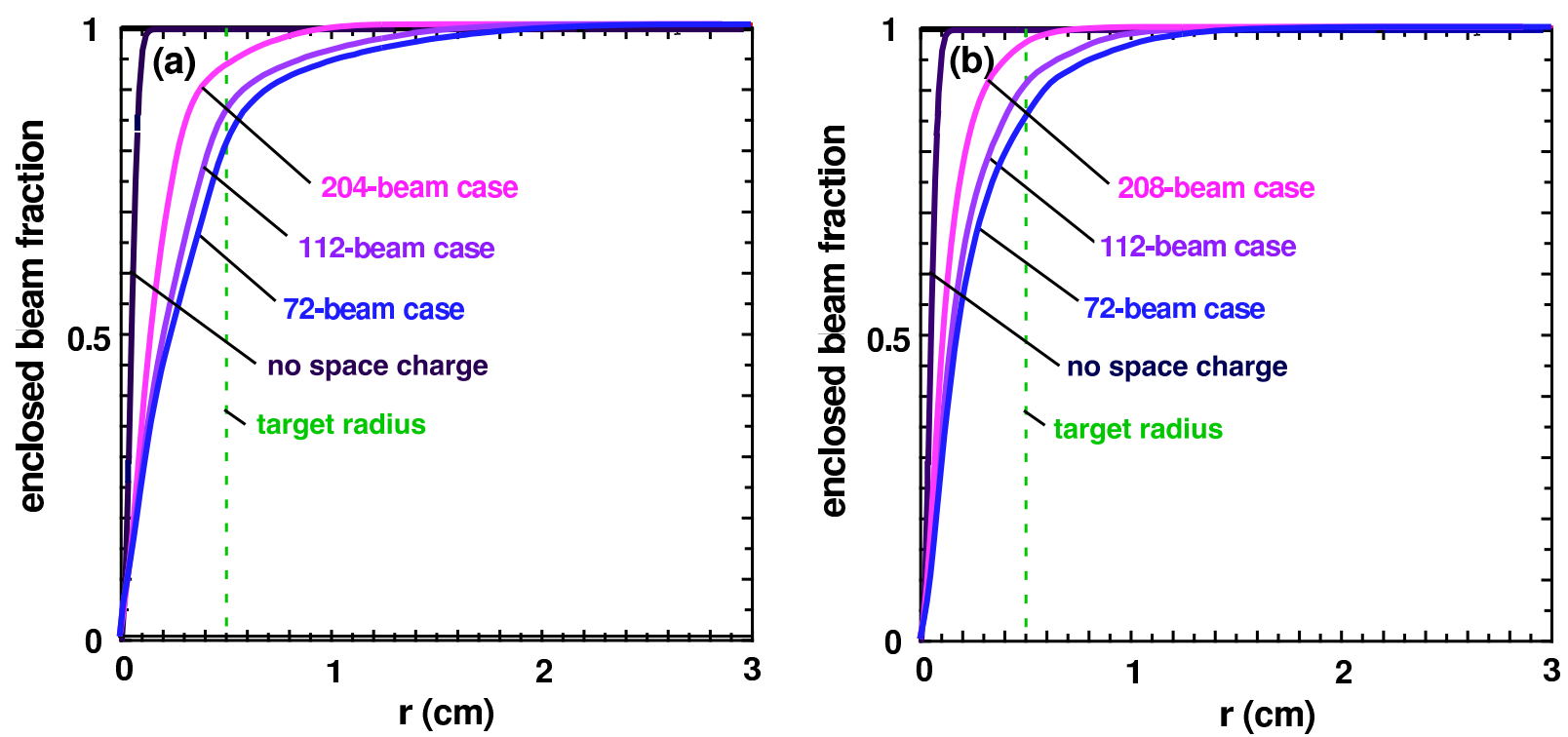

Figure 11: Enclosed fraction of beam ions with an electrically isolated plasma placed near the beam entrance in a backgroundgas density of (a) $5 \times 10^{13} \mathrm{~cm}^{-3}$ and (b) $7 \times 10^{12} \mathrm{~cm}^{-3}$, shown as a functions of radius for the three beam layouts treated here. Also, the enclosed-current fraction is shown for the same initial conditions but no space charge.
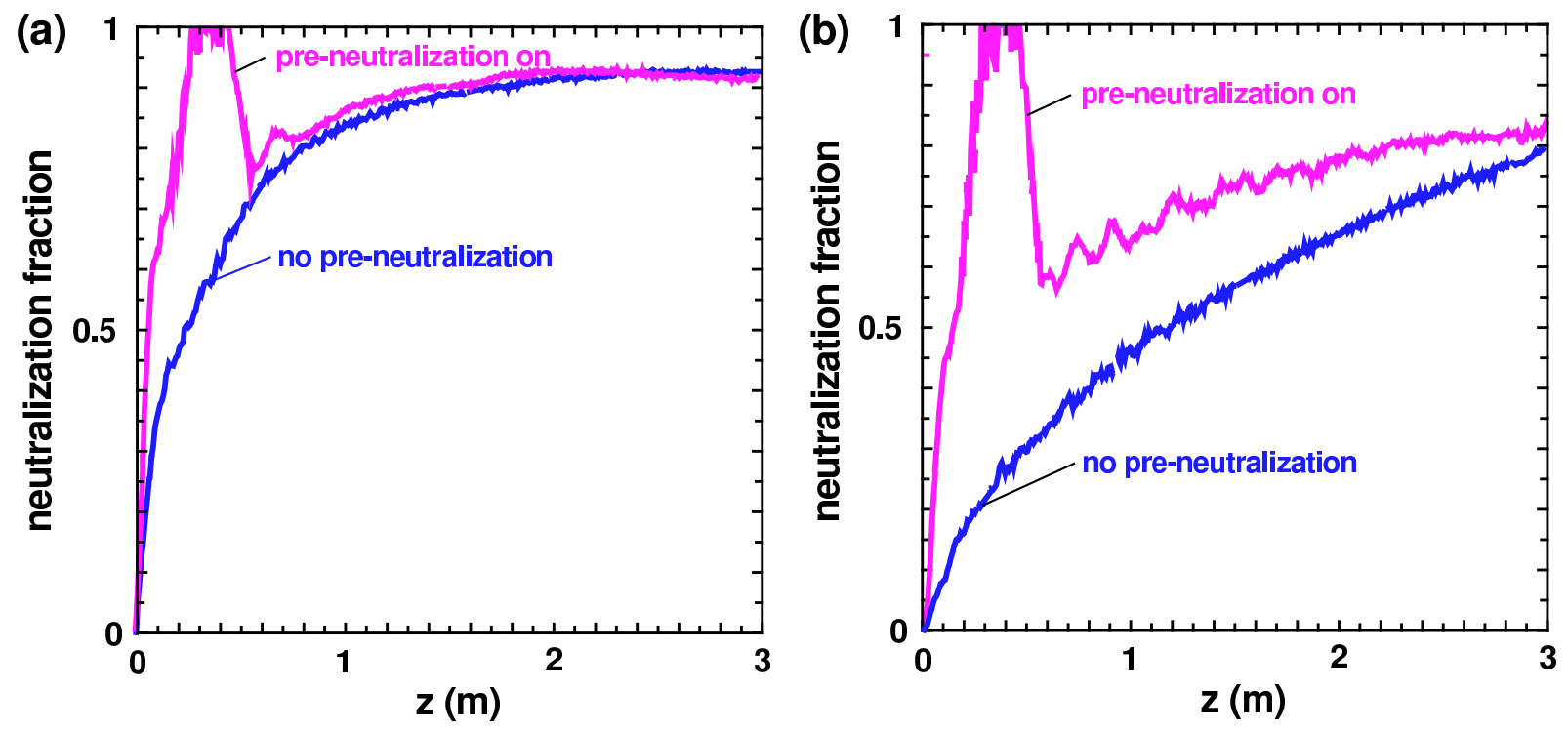

Figure 12: Mid-pulse neutralization fraction of a 651-A beam with and without a pre-neutralizing plasma, shown as a function of axial position $z$ for background-gas densities of (a) $5 \times 10^{13} \mathrm{~cm}^{-3}$ and (b) $7 \times 10^{12} \mathrm{~cm}^{-3}$.

with $35 \%$ without. Provided that the mean-free path for gas ionization exceeds the distance between the plasma and the target, as it does here, the additional neutralization picked up from the plasma persists until the ion beam reaches the target location, leading to a smaller net electric field within the beam and less emittance growth.

We have made a series of runs using the same range of gas densities used in Fig. 10 but adding an electrically isolated $10^{12} \mathrm{~cm}^{-3}$ pre-neutralizing plasma. As before, a current of $651 \mathrm{~A}$ is used, although analogous results are found for the other currents used here. The enclosed-fraction radii are plotted in Fig. 13, with smooth lines again drawn through the data points. Comparing the enclosed-fraction radii in the two figures, we see that pre-neutralization reduces the minimum waist radius by about $30 \%$ and effectively eliminates the density dependence of the focal spot for densities below about $1.5 \times 10^{13} \mathrm{~cm}^{-3}$.

The results here are insensitive to the density of the plasma and to its radial extent, so long as the number of plasma 

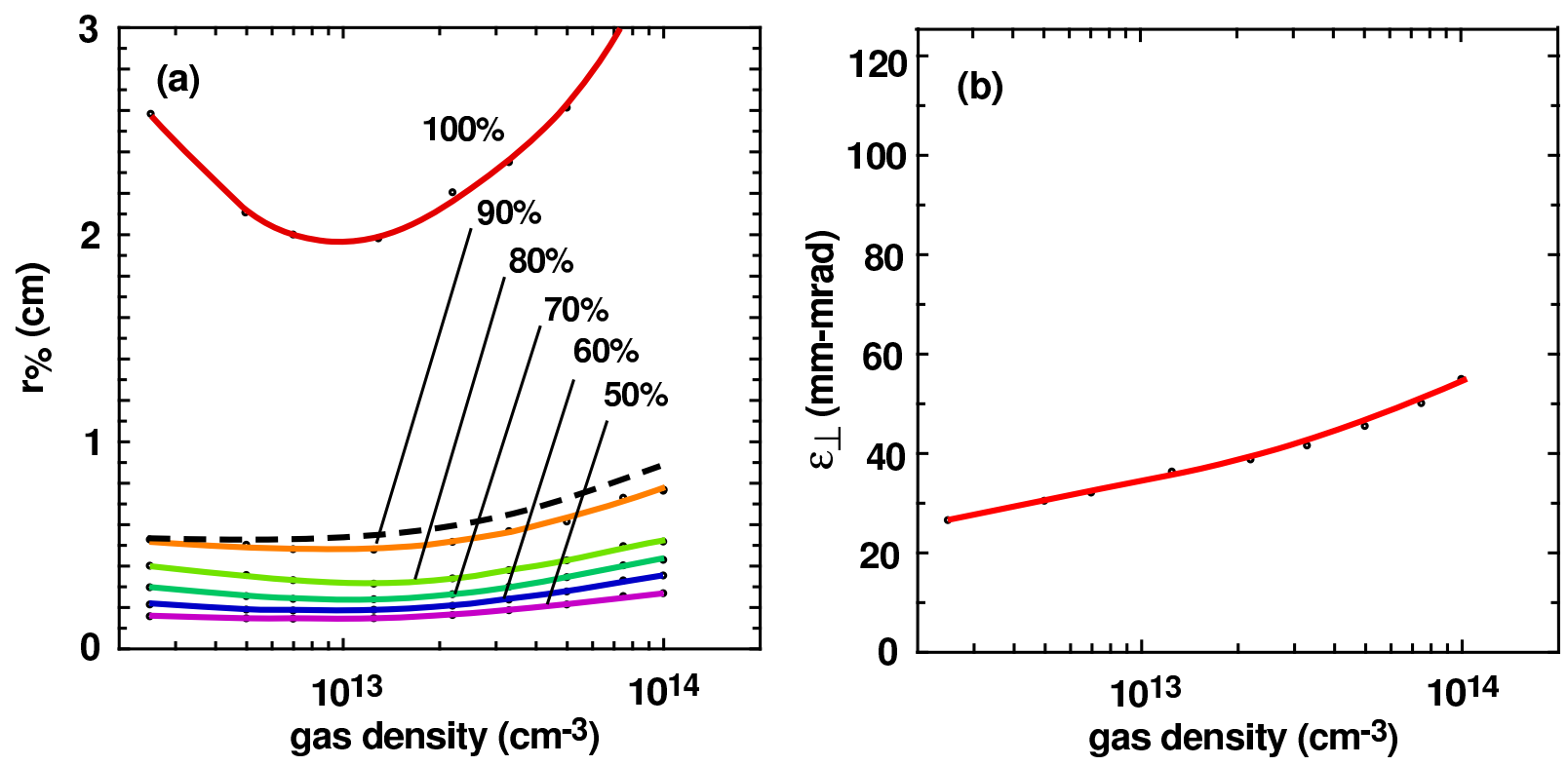

Figure 13: Radii enclosing specified percentages of beam ions at the beam waist with a pre-neutralizing plasma, shown as functions of the background-gas density. The dashed curve give the effective edge radius $R$.

electrons within the beam radius substantially exceeds the initial beam charge. Virtually no difference is seen between cases with densities of $10^{11} \mathrm{~cm}^{-3}$ and $10^{12} \mathrm{~cm}^{-3}$, but reducing the density to $10^{10} \mathrm{~cm}^{-3}$, which is slightly below the beam density at the same axial location, results in reduced neutralization and a $20 \%$ larger focal spot.

The main limitation of this work is the electrical isolation of the pre-neutralizing plasma. In the calculation, any electron pulled from the plasma by a passing beam leaves a fixed positive charge at its original position that retards the escape of other electrons. In practice, however, pre-neutralization would be done in the beam ports just upstream from the fusion chamber, so the plasma would be in electrical contact with a grounded metal wall. In this case, electrons would be drawn off the wall to maintain the quasineutrality of the plasma. We expect that this additional electron source will substantially increase the number of electrons captured by ion beam. Initial work by Rose et al.[19] supports this expectation, and more recent simulations by Welch et al.[20] indicate that the focal radius can be a factor of two smaller than similar cases with an electrically isolated plasma.

\section{E. Effects of molten-salt jets}

The principal omission from the foot-pulse simulations here is the absence of the molten-salt jets planned for wall protection. As presently conceived, a crisscrossed lattice of these jets would fill the outer two meters of the chamber ends, with aligned holes between jets for the beams. Each jet is expected to be a few centimeters in diameter, probably with millimeter-scale ripples. Such jets have several features that complicate realistic modeling: their low but non-negligible conductivity, their intrinsically three-dimensional geometry, and the likelihood that their surface will be ionized by ions from the beam halo and, after initial target heating, by $\mathrm{X}$ rays from the hohlraum ends.

To get a qualitative idea of how these jets might affect chamber transport of a foot pulse, we have studied an extremely crude computational model of the jets. Because BICrz is an axisymmetric code, the rectangular lattice of jets is replaced in the model by rings. For the first $2 \mathrm{~m}$ of chamber transport, the beam is circled by rings representing the jets, placed $5 \mathrm{~mm}$ outside the nominal beam radius and spaced 10-cm apart longitudinally. Due to the relatively coarse axial gridding, however, the rings are fewer in number than the planned jets, larger in cross-sectional area, and rectangular rather than circular in cross section, with a $10-\mathrm{cm}$ length and a 1-cm thickness. Perhaps the crudest aspect of the model is its representation of the molten salt by a plasma with anchored ions. Although this model is arguably adequate after the flibe surface has become ionized, it misrepresents the electrical properties of the flibe and ignores the work function that binds electrons to the salt.

The principal effect of the plasma rings is to contribute electrons to the passing beam. Some electrons are pulled transversely into the beam by the unneutralized space-charge field, but they have a larger transverse temperature than 

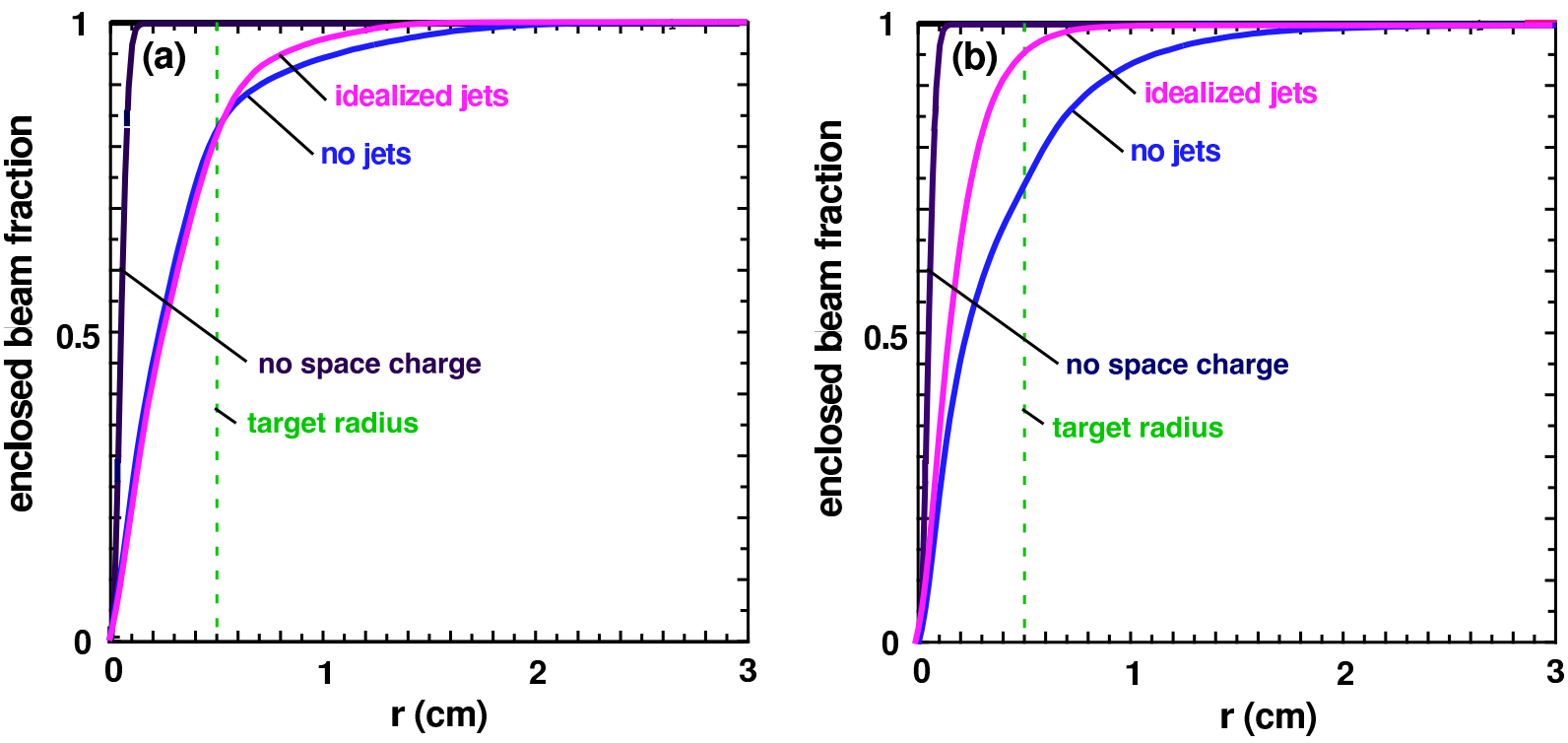

Figure 14: Enclosed fraction of beam ions with and without idealized flibe jets around the beam for the first $2 \mathrm{~m}$ of chamber transport through a background-gas density of (a) $5 \times 10^{13} \mathrm{~cm}^{-3}$ and (b) $7 \times 10^{12} \mathrm{~cm}^{-3}$, shown as a functions of radius. Also, the enclosed-current fraction is shown for the same initial conditions but no space charge. Nominal parameters for the 112-beam layout are used for all cases here.

electrons from a pre-neutralizing plasma, so they are more readily lost during transport, being replaced by electrons from other rings. The net effect is seen in Fig. 14, which shows results for a $651-\mathrm{A}$ beam of $3-\mathrm{GeV} \mathrm{Pb}^{+1}$ ions. At the nominal $5 \times 10^{13} \mathrm{~cm}^{-3}$ background-gas density, shown in Fig. 14a, the rings slightly reduce the radius of ions in the beam halo but have a negligible effect on the beam core. This limited effect is due in part to the effective neutralization provided by collisional ionization. In addition, a comparison of beam neutralization for the cases with and without plasma rings shows that the small additional neutralization picked up from the rings is lost during the final meter of transport. The rings are much more effective at neutralizing the beam for a background density of 7 $\mathrm{x} 10^{12} \mathrm{~cm}^{-3}$, as seen in Fig. 14b. Due to the slow rate of collisional ionization, neutralizing electrons from the rings cause a substantial reduction in the net space charge, increasing the fraction of beam ions hitting the target from $75 \%$ to over $95 \%$. Even though electrons from the rings are lost more readily that those from a plasma, the focal spot for this case is smaller than the corresponding 112-beam case in Fig. 11b because the electrons only have to remain in the beam for the final meter, compared with $2.5 \mathrm{~m}$ for the case with a plasma.

Despite the obvious shortcomings of the model, the simulations suggest that the rings could be a useful source of electrons, provided that their surface is sufficiently ionized by halo particles from the beam or, later, by target $\mathrm{x}$ rays. We should note, however, that this axisymmetric calculation necessarily ignores the asymmetry of actual flibe jets, which could affect the transverse position or possibly the stability of the beam. Three-dimensional simulations using a more plausible model of the molten-salt jets are needed before we can claim to understand this aspect of chamber transport.

\section{CONCLUSIONS}

The preliminary simulations reported here suggest that transport of foot pulses in a fusion chamber poses several challenges. Although these pulses have a lower current and energy than main pulses, they must reach the target and do the initial hohlraum heating without the benefit of the photoionized background-gas plasma that surrounds the target when the main pulses arrive. Consequently, foot pulses are more poorly neutralized in their final stages of radial compression, leading to emittance growth and a poor focus. These problems can be reduced by using more foot pulses, but only at the cost of greater complexity.

The use of an electrically isolated pre-neutralizing plasma reduces the focal spot size, but the effect is limited at higher background-gas density by the high degree of neutralization that results from collisional ionization. If the background-gas density is as low as recent work suggests, then pre-neutralization is found to work substantially better 
and may allow an acceptable focal spot for 112 or perhaps even 72 foot beams. These cases, of course, must be rerun with a realistic target model, flibe jets, and photoionization before we can feel confident that foot pulses can be successfully transported to an HIF target.

\section{Acknowledgments}

Work performed under the auspices of the US Department of Energy by University of California Lawrence Livermore National Laboratory and Lawrence Berkeley National Laboratory under Contracts No. W-7405-ENG-48 and DE-AC3-76SF00098.

[1] J. Lindl, Plas. Physics 2, 3933 (1995).

[2] M. Tabak and D. A. Callahan-Miller, Nucl. Instr. and Meth in Phys. Res. A 415, 75 (1998).

[3] D. A. Callahan-Miller and M. Tabak, Nucl. Fusion 39, 1547 (1999).

[4] D. A. Callahan, Fusion Eng. and Design 32-33, 441 (1995).

[5] N. Barboza, Fusion Eng. and Design 32-33, 453 (1995).

[6] W. M. Sharp, D. A. Callahan-Miller, A. B. Langdon, M. S. Armel, and J.-L. Vay, Nucl. Instr. and Meth in Phys. Res. A 464, 284 (2001).

[7] J. D. Lawson, J. Electron. Control 5, 146 (1958).

[8] C. K. Birdsall and A. B. Langdon, Plasma Physics via Computer Simulation (McGraw-Hill, New York, 1988).

[9] A. B. Langdon, Bull. Am. Phys. Soc. 35, 2415 (1991).

[10] A. B. Langdon, Comput. Phys. Commun. 70, 447 (1992).

[11] P. F. Dubois, "The Basis System," LLNL Doc. No. M-225 (1988).

[12] R. E. Olson, Nucl. Instr. and Meth in Phys. Res. A 464, 93 (2001).

[13] M. S. Armel, "Atomic Processes for Heavy Ion Inertial Fusion," Ph. D. Thesis, University of California Berkeley (2000).

[14] R. W. Moir, R. L. Bieri, X. M. Chen, T. J. Dolan, M. A. Hoffman, P. A. House, R. L. Leber, J. D. Lee, Y. T. Lee, J. C. Shrock, M. T. Tobin, and W. H. Williams, Fusion Technol. 25, 5 (1994).

[15] D. R. Olander, G. Fukuda, and C. F. Baes Jr, Fusion Science Technol. 41, 141 (2002).

[16] C. L. Olson, "HIF Transport Issues for $P>10^{-3}$ Torr and $Z>1$," Proc. of the 1986 HIF Symposium, Washington, DC, AIP Conf. Proc. 152, 215 (1986).

[17] E. P. Lee, S. S. Yu, and W. A. Barletta, Nucl. Fusion 21, 8 (1981).

[18] P. C. Efthimion and R. C. Davidson, Nucl. Instr. and Meth in Phys. Res. A 464, 310 (2001).

[19] D. V. Rose, D. R. Welch, B. V. Oliver, W. M. Sharp, and A. Friedman, Nucl. Instr. and Meth in Phys. Res. A 464, 299 (2001).

[20] D. R. Welch, D. V. Rose, W. M. Sharp, and S. S. Yu, "Effects of Pre-Neutralization on Heavy-Ion-Fusion Chamber Transport," to be published in Laser \& Part. Beams. 